\title{
Minute primary extramedullary plasmacytomas of the large intestine
}

An 84-year-old woman underwent a follow-up colonoscopy 4 years after undergoing endoscopic mucosal resection (EMR) for colonic adenoma. Two small polyps were found in the cecum and the rectum ( $\bullet$ Fig. 1 a,b) and EMR was performed.

Histologically, both resected specimens showed a dense infiltration of plasma cells ( $\bullet$ Fig. 2 a, b).

They were considered to be monoclonal neoplastic cells because they were immunohistochemically positive for kappa light chains but negative for lambda light chains ( $\bullet$ Fig. 3a,b).

A subsequent skeletal survey and bone marrow aspirate biopsy were normal. No monoclonal paraprotein was identified in the serum or urine, and there was no hypercalcemia or renal failure. Upper gastrointestinal endoscopy showed atrophic gastritis only. Dynamic computed tomography showed no lesion associated with the plasmacytoma. On the basis of these results, a diagnosis of primary extramedullary plasmacytomas (PEMPs) of the cecum and rectum was made.

The diagnosis of PEMP requires demonstration of a monoclonal plasma cell infiltration without evidence of multiple myeloma [1,2]. Approximately $10 \%$ of PEMPs are found in the gastrointestinal tract $[3,4]$. PEMP of the large intestine is extremely rare, and to the best of our knowledge, only 13 previous cases of PEMP of the large intestine have been reported in the English literature. All of these previous cases were symptomatic, advanced, single tumors, which underwent surgical treatment, except for one case which was resected using EMR [5]. Tumors in these cases have no special endoscopic appearance, and only one endoscopic image has been published [5]. In our case, we described two minute PEMPs (cecum $=4 \mathrm{~mm}$ in diameter; rectum $=2 \mathrm{~mm}$ in diameter) without symptoms in the large intestine with clear endoscopic images. To our knowledge, there are no similar previous reports.

In conclusion, our case is very important for the understanding of the early morphology of PEMP in the large intestine.

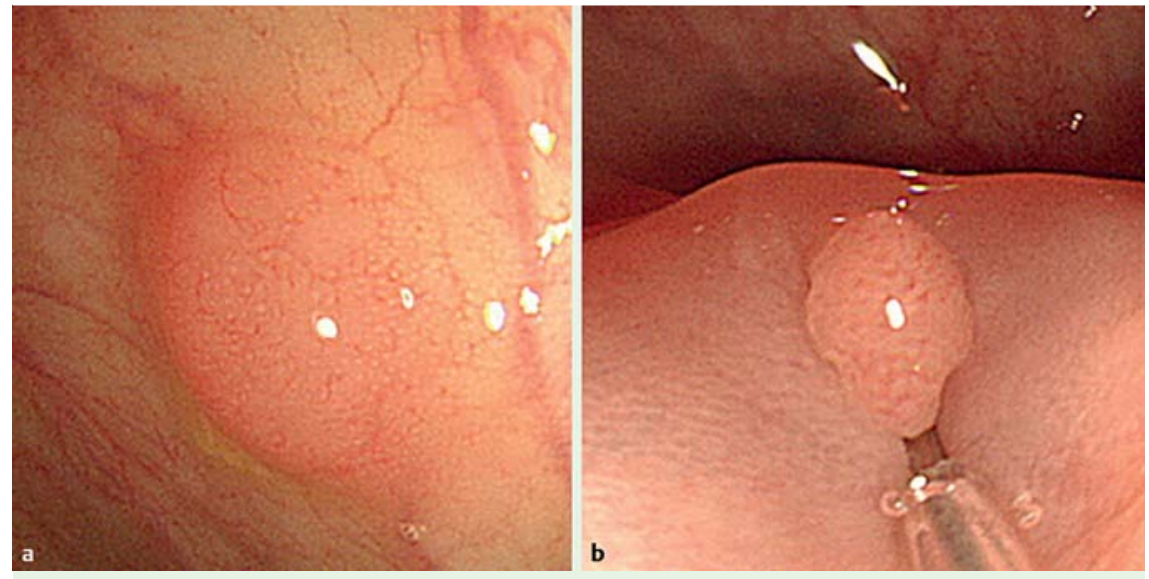

Fig. 1 Endoscopic appearance of primary extramedullary plasmacytomas. a In the cecum, the slightly reddish sessile polyp with a normal surface was suggestive of a submucosal tumor. $\mathbf{b}$ In the rectum, the mucosal surface of the tumor was different from the surrounding normal mucosa, suggesting an epithelial tumor.

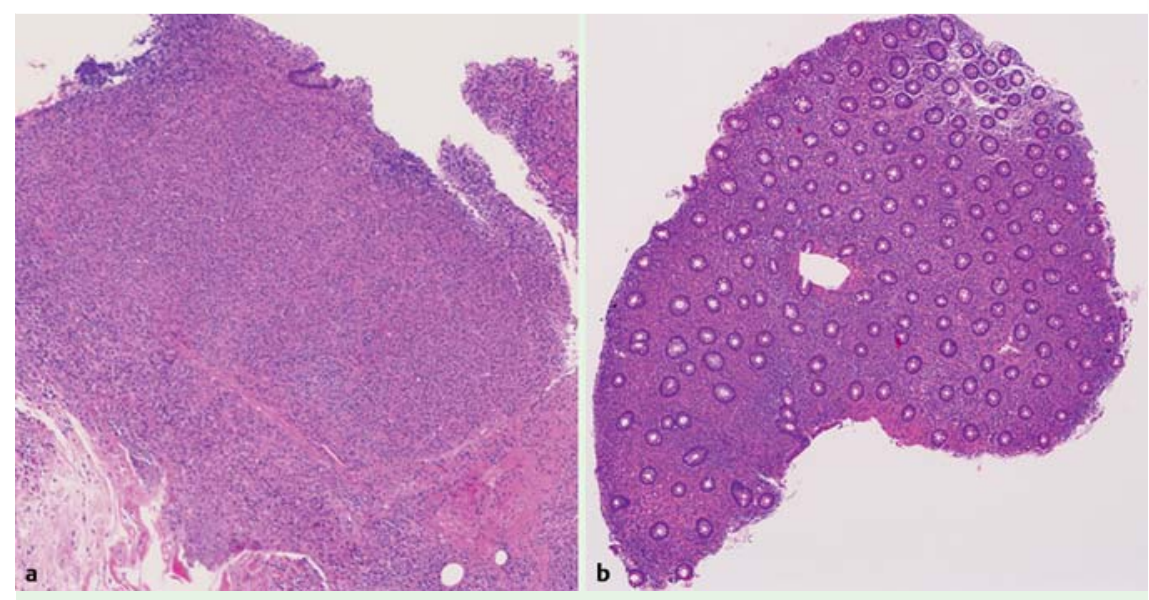

Fig. 2 Low-power view of primary extramedullary plasmacytomas: a cecal; b rectal.

Endoscopy_UCTN_Code_CCL_1AD_2AC

\section{Competing interests: None}

Y. Nakagawa ${ }^{1}$, T. Nagai ${ }^{1}$, H. Okawara ${ }^{1}$, H. Nakashima ${ }^{1}$, A. Hisamatsu ${ }^{1}$, M. Syutou $^{1}$, M. Yamauchi ${ }^{1}$, S. Kai ${ }^{2}$, T. Nakaya$\mathrm{ma}^{3}$, S. Yokoyama ${ }^{4}$, K. Murakami ${ }^{5}$,

\section{T. Fujioka ${ }^{5}$}

Department of Gastroenterology, Oita Kouseiren Tsurumi Hospital, Beppu, Japan

2 Department of Pathology, Oita Kouseiren Tsurumi Hospital, Beppu, Japan

3 Department of Hematology, Oita Kouseiren Tsurumi Hospital, Beppu, Japan
4 Department of Pathology, Faculty of Medicine, Oita University, Yufu, Japan

5 Department of General Medicine and Gastroenterology, Faculty of Medicine, Oita University, Yufu, Japan

\section{References}

1 Schweers CA, Shaw MT, Nordquist RE et al. Solitary cecal plasmacytoma: electron microscopic, immunologic, and cytochemical studies. Cancer 1976; 37: 2220-2223

2 Rygaard-Olsen C, Boedker A, Emus HC et al. Extramedullary plasmacytoma of the small intestine. A case report studied with electron microscopy and immunoperoxidase technique. Cancer 1982; 50: 573-576

3 Hampton JM, Gandy JR. Plasmacytoma of the gastrointestinal tract. Ann Surg 1957; 145: $415-422$ 

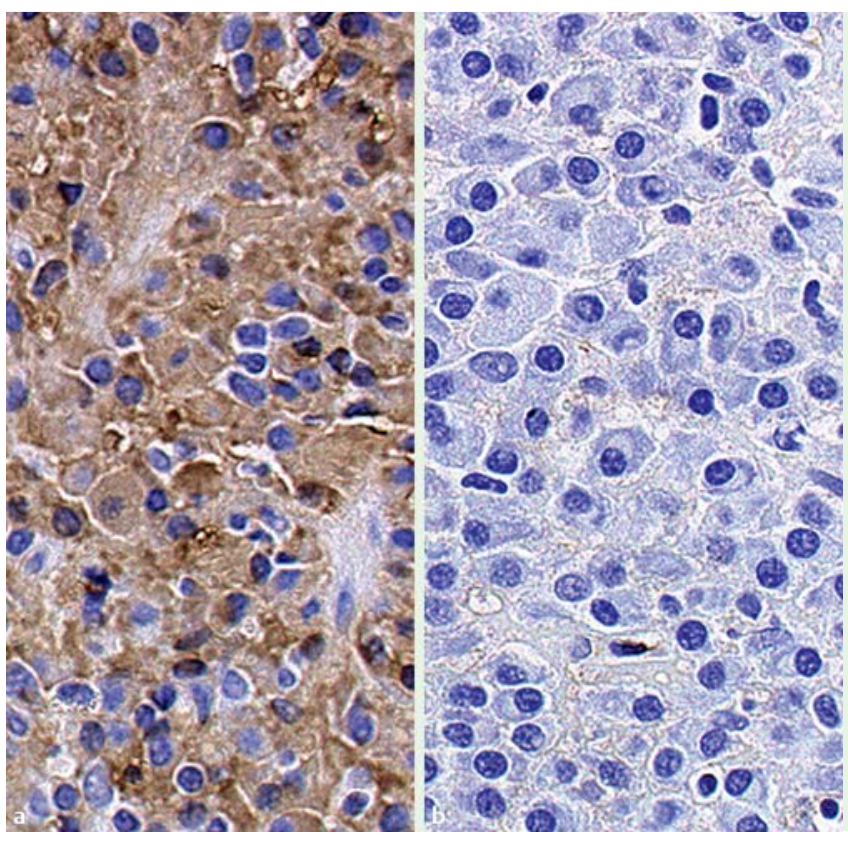

Fig. 3 Immunohistochemical staining for primary extramedullary plasmacytomas: a kappa light chains; b lambda light chains.
4 Asselah F, Crow J, Slavin G et al. Primary plasmacytoma of the small intestine. Histopathology 1982; 6: 631-645

5 Hashiguchi K, Iwai A, Inoue T et al. Extramedullary plasmacytoma of the rectum arising in ulcerative colitis: case report and review. Gastrointest Endosc 2004; 59: $304-$ 307

\section{Bibliography}

DOI $10.1055 / \mathrm{s}-0030-1256138$

Endoscopy 2011; 43: E105-E106

(c) Georg Thieme Verlag KG Stuttgart · New York . ISSN 0013-726X

\section{Corresponding author} Y. Nakagawa, MD

Department of Gastroenterology Oita Kouseiren Tsurumi Hospital

Tsurumi, 4333

Beppu 874-8585

Japan

Fax: +81-977-237039

nakagawa4423@ybb.ne.jp 